\title{
MedienPädagogik
}

Zeitschrift für Theorie und Praxis der Medienbildung www.medienpaed.com

Themenheft Nr. 25: Medienbildung und informatische Bildung - quo vadis? Hrsg. von Klaus Rummler, Beat Döbeli Honegger, Heinz Moser und Horst Niesyto

\section{Zur Relevanz informatischer Bildung in der Schule für den Erwerb computer- und informationsbezogener Kompetenzen als Teilaspekt von Medienbildung Ergebnisse für Deutschland und die Schweiz im internationalen Vergleich}

Birgit Eickelmann und Kerstin Drossel

\section{Zusammenfassung}

Bisher liegen kaum empirische Studien vor, die das Verhältnis zwischen Medienbildung und informatischer Bildung untersuchen. Auf der Datengrundlage der Schulleistungsstudie ICILS 2013 (International Computer and Information Literacy Study), die erstmals international vergleichend computer- und informationsbezogene Kompetenzen von Achtklässlerinnen und Achtklässlern in weltweit 21 Bildungssystemen untersucht hat, beleuchtet der vorliegende Beitrag die vorgenannte Schnittstelle in Deutschland und in der Schweiz. Daran anknüpfend, dass die in der Studie mit computerbasierten Tests erfassten Kompetenzen einerseits als fächerübergreifende Schlüsselkompetenzen konzipiert sind, ihnen aber andererseits vielfach eine Affinität zu informatischer Bildung zugeschrieben wird, werden deskriptive und regressionsanalytische Sekundäranalysen präsentiert.

Es zeigt sich für beide Länder, dass Schülerinnen und Schüler, die das Fach Informatik belegen, im Mittel deutlich geringere computer- und informationsbezogene Kompetenzen erreichen als die gleichaltrige Gruppe, die das Fach nicht belegt. Nutzen die Jugendlichen, die Informatik belegen, allerdings einen Computer im Informatikunterricht (oder einem Fach zur informatischen Bildung), schneiden sie im Mittel besser ab. Diese Befundlage bleibt bestehen, wenn man die Angaben der Jugendlichen hinsichtlich der in der Schule erlernten computerbezogenen Fähigkeiten sowie ihre Motivation und Einstellungen zur Computernutzung hinzuzieht und auch für das Geschlecht der Jugendlichen sowie für das kulturelle Kapital der Familien kontrolliert. 


\title{
On the Relevance of IT Education at School for the Purpose of Acquiring Computer and Information Literacy as part of Media Education - Results from Germany and Switzerland on a Level of International Comparison
}

\begin{abstract}
Thus far, only few empirical studies have been conducted that examine the relationship between media education and IT education. On the basis of data from the large-scale comparative study of ICILS 2013 (International Computer and Information Literacy Study) on educational achievement that for the first time compares computer and information literacy skills of Grade 8 students from 21 educational systems around the world, the present contribution sheds light on the previously mentioned interface with respect to Germany and Switzerland. Building on the fact that the student competencies - measured in ICILS 2013 by means of computer-based testing - are conceived as interdisciplinary key competencies on the one hand but often show an affinity to IT education on the other hand, descriptive and regressive secondary analyses are presented. For both Germany and Switzerland, it could be shown that students who attend IT classes demonstrate significantly lower levels of computer and information literacy than their peers who have not taken IT classes. However, if students who attend such classes use a computer in them (or in a similar subject related to IT education), their achievement is - on average - higher. This finding remains in place if the students' answers regarding the computer-related skills acquired at school as well as their motivation and attitudes towards computer usage are included. The same is true when controlling for the students' gender and their families' cultural capital.
\end{abstract}

\section{Einleitung}

Während in den letzten Jahren zunächst Forschungen und Arbeiten zur Medienbildung sowie die Medienpädagogik und der Bereich der informatischen Bildung und der Fachdidaktik Informatik grösstenteils noch unverbunden nebeneinander standen, ist in letzter Zeit zu beobachten, dass sich diese Disziplinen in ihren Aktivitäten und auch in der Forschung und Theoriebildung - und zudem in sehr konkreten Kooperationen und gemeinsamen Erklärungen - annähern (vgl. u. a. GI-Gesellschaft für Informatik, 2016). Dies erscheint vor dem Hintergrund der Digitalisierung aller Lebens- und Arbeitsbereiche zunehmend notwendig und spiegelt sich auch in den bisher nur in Ansätzen genutzten Synergien wider, die die Teilbereiche miteinander verbinden. Eine wichtige Teilfrage, die auch eine relevante treibende Kraft für zukünftige Entwicklungen sein könnte, ist die Frage, wie der schulische Kompetenzerwerb - sowohl in Bezug auf die informatische Bildung als auch im Bereich der Medienbildung - von den Disziplinen profitieren kann und an welchen 
Stellen sich Überschneidungsbereiche oder Anknüpfungspunkte ausmachen lassen. Um zu dieser Fragestellung Antworten zu finden, die auch die Grundlage für die Ableitung von zukünftigen Entwicklungsperspektiven sein könnten, bedarf es neben theoretischen Konzepten auch einer empirischen Grundlage, die bisher in weiten Teilen noch nicht bzw. nur in Ansätzen vorliegt.

An dieser Stelle setzt der vorliegende, empirisch ausgerichtete Beitrag an und nutzt dazu die Datengrundlage der ICILS-2013-Studie (International Computer and Information Literacy Study; Laufzeit 2010-2014). Mit dieser international vergleichenden Schulleistungsstudie, die von der IEA (International Association for the Evaluation of Educational Achievement) koordiniert wurde, liegen erstmalig empirisch gesicherte Erkenntnisse über den Kompetenzstand von Achtklässlerinnen und Achtklässlern im Bereich der computer- und informationsbezogenen Kompetenzen vor (Bos et al. 2014; Fraillon et al. 2014; Konsortium icils.ch 2015). Wenngleich der betrachtete Kompetenzbereich, der auf internationaler Ebene computer and information literacy (CIL) heisst, als fächerübergreifende Schlüsselkompetenz definiert ist und mit Bezug auf den Bereich der neuen Technologien hohe Affinitäten zu dem im deutschsprachigen Raum genutzten Medienkompetenzkonzept hat (Bos, Eickelmann \& Gerick, 2014; vgl. dazu weiterhin bereits Aufenanger 1997; Schulz-Zander 1998; Tulodziecki 1997), eröffnet diese Studie Möglichkeiten, Verknüpfungen zur informatischen Bildung herzustellen. In der repräsentativen Stichprobe liegen über die reinen Testdaten im Bereich der computerund informationsbezogenen Kompetenzen auch Daten zu Rahmenbedingungen des Erwerbs dieser Kompetenzen vor, zu denen u. a. die Fragestellung gehört, ob die Schülerinnen und Schüler das Fach Informatik belegen und ob bzw. wie häufig sie im Informatikunterricht Computer nutzen. Da an der Studie sowohl Deutschland als auch die Schweiz teilgenommen haben, eröffnet sich für beide Länder mit den in diesem Beitrag aufbereiteten Analysen die Möglichkeit, der Frage nach der Bedeutung des Faches Informatik zum Kompetenzerwerb im Umgang mit neuen Technologien und digitalen Informationen nachzugehen. Dies ist abgesehen von dem Beitrag zur oben beschriebenen aktuellen Diskussion auch vor dem Hintergrund bedeutsam, als dass die Studie gezeigt hat, dass Achtklässlerinnen und Achtklässler sowohl in Deutschland als auch in der Schweiz im internationalen Vergleich nur über mittlere durchschnittliche computer- und informationsbezogene Kompetenzen verfügen und die Wirksamkeit schulischer Bildung zum Erwerb dieser Kompetenzen in beiden Ländern bisher eher von begrenzter Reichweite ist (vgl. Bos et al. 2014). Eine besondere Rolle wird seit der Veröffentlichung der Ergebnisse der Studie in der öffentlichen und bildungspolitischen Diskussion jedoch der informatischen Bildung und damit insbesondere dem Fach Informatik zugeschrieben, da dieser Bereich auf den ersten Blick eine besondere Affinität zum Bereich des kompetenten Umgangs mit neuen Technologien und digitalen 
Informationen hat. Wenngleich der kompetente Umgang mit neuen Technologien und digitalen Informationen, der im Fokus der ICILS-Studie steht, nicht unmittelbar mit den Formulierungen in den Curricula des Informatikunterrichts adressiert wird, zeigen sich theoretisch-konzeptionell vor allem Überschneidungen zwischen dem mit der Studie erfassten Konstrukt und den von der Informatik als eigene Disziplin abzugrenzenden Bereich der digital literacies (Gander et al. 2013). Sehr allgemein formuliert besteht bereits seit dem Jahr 2008 eine Intention der Grundsätze und Standards für die Informatik in der Schule darin, dass «alle Schülerinnen und Schüler künftig den Einsatz von Computern und Informations- und Kommunikationstechnik zu ihrem Nutzen bewältigen können [sollen]» (Gesellschaft für Informatik 2008, 2). Für die Schweiz hat in diesem Kontext die D-EDK (Deutschschweizer Erziehungsdirektorenkonferenz) im Jahr 2015 in ihrem Schlussbericht zum Lehrplan 21 den Bereich «Medien und Informatik» gemeinsam ausgewiesen und beide Bereiche miteinander explizit verbunden und neu ausgerichtet (vgl. D-EDK 2015), wobei drei Kompetenzbereiche «Anwenden, Medien und Informatik» unterschieden werden (vgl. Döbeli Honegger 2015). Weiterhin wurde in Deutschland auf nationaler Ebene im Sommer 2015 ein gemeinsamer Antrag der Regierungsparteien zur Stärkung der digitalen Bildung und Förderung von Medienkompetenz verabschiedet, der nicht nur explizit Bezug zu den Ergebnissen der Studie ICILS 2013 nimmt, sondern auch auf die Stärkung des Unterrichtsfachs Informatik eingeht (vgl. Deutscher Bundestag 2015). Dem skizzierten Anliegen folgend und empirisch prüfend, untersucht der vorliegende Beitrag nunmehr diese Schnittstelle und nutzt dafür die Deutsche und Schweizer Datengrundlage der Studie ICILS 2013. Bei den nachfolgenden Ausführungen und Analysen handelt es sich also um Sekundäranalysen zur Studie ICILS 2013, deren Anlage nicht primär auf die Untersuchung dieser Schnittstelle abzielte. Dennoch bietet die Studie anhand der vorliegenden Daten und Instrumente die Möglichkeit, zumindest in einem begrenzten Ausschnitt den Zusammenhang zwischen dem Erwerb computer- und informationsbezogener Kompetenzen und den in der Studie erfassten Aspekten zum Informatikunterricht zu untersuchen.

Den Analysen voran, wird in einem ersten Schritt in dem Beitrag zur Verortung der hier vorgestellten Forschungsarbeit zunächst das Konzept der computer- und informationsbezogenen Kompetenzen sowie das Abschneiden der Schweiz und Deutschlands im internationalen Vergleich zu zentralen Studienergebnissen vorgestellt (für eine ausführlichere Ergebnisdarstellung vgl. u. a. Bos et al. 2014; Fraillon et al. 2014; Senkbeil et al. 2014). Im Anschluss daran werden entlang von vier Forschungsfragen das Verhältnis von Medienbildung, hier fokussiert auf den als Teilaspekt zu verstehenden Bereich der computer- und informationsbezogenen Kompetenzen, und informatischer Bildung im Rahmen der begrenzten, aber dennoch informativen Möglichkeiten, die die Studie bietet, untersucht. Zunächst wird 
betrachtet, ob Schülerinnen und Schüler der achten Jahrgangsstufe, die das Fach Informatik belegen, über höhere computer- und informationsbezogene Kompetenzen verfügen, als solche, die das Fach nicht belegen. In einem zweiten Schritt wird daran anknüpfend untersucht, ob Jugendliche, die das Fach Informatik belegen, häufiger angeben, computerbezogene Fähigkeiten in der Schule erlernt zu haben, als solche, die das Fach nicht belegen. Weiterhin wird in den Blick genommen, ob sich die betrachteten Gruppen hinsichtlich der Motivation und bezogen auf die Einstellungen in Bezug auf das Nutzen und das Lernen mit dem Computer unterscheiden. Für alle drei Aspekte werden die Ergebnisse für Deutschland und die Schweiz innerhalb eines europäischen und internationalen Vergleichs verortet und es wird jeweils vertiefend betrachtet, ob die unterrichtliche Computernutzung zur besseren Beschreibung und Deutung von Unterschieden herangezogen werden kann. Im Hinblick auf den Erwerb computer- und informationsbezogener Kompetenzen ist dabei die theoretische Vorannahme relevant, dass die Nutzung von neuen Technologien selbst hohes Potenzial birgt, diesbezüglich Kenntnisse und Fähigkeiten zu entwickeln (Fletcher et al. 2012; Fraillon et al. 2013). In einem letzten Analyseschritt werden mit einer abschliessenden Forschungsfrage in einer Regressionsanalyse die Zusammenhänge vertiefend unter Kontrolle von Hintergrundmerkmalen untersucht. Abschliessend werden die gewonnenen Befunde im Kontext der Diskussion über die Schnittstelle zwischen Medienbildung und informatischer Bildung eingeordnet. Der Anlage einer internationalen Vergleichsstudie geschuldet, wird im Kontext der Analysen der Begriff «Informatikunterricht` verwendet, wenngleich in den deutschen Bundesländern und auch in den Schweizer Bildungsregionen unterschiedliche Begrifflichkeiten dafür verwendet werden und aus den internationalen Fragebögen die Fachbezeichnung «information technology/computer studies or similar» (Jung/Carstens 2015, 105) in den jeweiligen nationalen Kontext übersetzt wurde. Zu berücksichtigen ist im Verständnis aller durchgeführten Analysen sowie für die Interpretation der Befunde zudem, dass es sich bei ICILS 2013 um eine Studie mit einmaliger Erhebung und damit um ein querschnittliches Design handelt, sodass die durchgeführten Zusammenhangsanalysen keine Aussagen über Wirkungsrichtungen und Kausalzusammenhänge treffen können. 


\section{Das Konzept der computer- und informationsbezogenen Kompetenzen und das Abschneiden Deutschlands und der Schweiz im internationalen Ver- gleich in der Studie ICILS 2013}

Die International Computer and Information Literacy Study ICILS 2013 - Anlage, Konzeptionierung und zentrale Befunde für Deutschland und die Schweiz Um die nachfolgenden Analysen im Kontext der grösser angelegten Studie ICILS 2013 zu verorten, werden in diesem Abschnitt zunächst die Studie und ausgewählte Befunde für Deutschland und die Schweiz in gebotener Kürze vorgestellt.

Mit der Schulleistungsstudie ICILS 2013 der IEA wurde erstmalig computerbasiert und international vergleichend untersucht, über welche computer- und informationsbezogenen Kompetenzen (computer and information literacy, kurz: CIL) Schülerinnen und Schüler in der achten Jahrgangsstufe verfügen (Fraillon et al. 2014). An der Studie nahmen neben Deutschland und der Schweiz weltweit 19 weitere Bildungssysteme teil. Als Bildungsmonitoringstudie angelegt, liefert ICILS 2013 erstmalig zuverlässige empirische Befunde zu den Kompetenzständen von Jugendlichen im Umgang mit neuen Technologien und digitalen Informationen in den beteiligten Ländern im internationalen Vergleich (Eickelmann, Gerick u. Bos 2014). Zusätzlich zu den Kompetenzen wurden im Rahmen der Studie auch die Rahmenbedingungen, unter denen der Kompetenzerwerb stattfindet, untersucht. Dafür wurden Kontextfragebögen für Schülerinnen und Schüler, Lehrpersonen, Schulleitungen sowie IT-Koordinatorinnen und IT-Koordinatoren eingesetzt. Ein wichtiger Aspekt in diesem Zusammenhang ist dabei die schulische Nutzung neuer Technologien, die auch nach Unterrichtsfächern bzw. nach Fächergruppen ausgewiesen wird (vgl. Eickelmann, Schaumburg, et al. 2014) und somit auch Analysen für den Informatikbereich zulässt, die im vorliegenden Beitrag im Vordergrund stehen werden.

Unter dem in ICILS 2013 erfassten Konstrukt der computer- und informationsbezogenen Kompetenzen werden die «individuelle[n] Fähigkeiten einer Person [verstanden], die es ihr erlauben, Computer und neue Technologien zum Recherchieren, Gestalten und Kommunizieren von Informationen zu nutzen und diese zu bewerten, um am Leben im häuslichen Umfeld, in der Schule, am Arbeitsplatz und in der Gesellschaft erfolgreich teilzuhaben» (Eickelmann, Bos, et al. 2014, 45). Diese Kompetenzen werden über computerbasierte Tests gemessen und stehen unter der übergeordneten Fragestellung, welchen Beitrag der Informatikunterricht möglicherweise zum Erwerb dieser Kompetenzen leistet, im Fokus des vorliegenden Beitrages. Das Konstrukt der computer- und informationsbezogenen Kompetenzen, das den Kompetenztests zugrunde liegt, ist im theoretischen Ansatz der Studie in zwei Teilbereiche gegliedert, die wiederum jeweils in Aspekte 
differenziert werden (Senkbeil et al. 2014). Teilbereich I «Informationen sammeln und organisieren> stellt die rezeptive Komponente dar und beinhaltet drei Aspekte, von denen der erste Aspekt auf grundlegende Kompetenzen im Umgang mit Computern abhebt, wie beispielsweise das Öffnen und Speichern von Dateien oder das Erkennen eines Dateityps anhand seiner Dateiendung (vgl. ebd.). Der zweite Aspekt umfasst Fähigkeiten wie das Finden und Bewerten von Informationen. Im Einzelnen geht es vor dem Hintergrund der beständig anwachsenden Fülle von Informationen, die über das Internet bereitgestellt wird, um die Fertigkeit, Informationen zu lokalisieren, zu identifizieren, sie abzurufen, zu filtern und zu speichern. Der dritte Aspekt dieses Teilbereichs fokussiert ergänzend das Verarbeiten und Organisieren von Informationen. Er umfasst die Fähigkeit, Informationen aufzubereiten, zu organisieren und zu speichern, sodass diese effizient für spezifische Fragestellungen genutzt werden können. Anschaulich gehören hierzu beispielsweise Kenntnisse über das kriteriengeleitete Sortieren und Filtern von Informationen aus einer Datenbank oder das Erstellen einer Dateistruktur innerhalb eines Verzeichnisbaums. Teilbereich II 〈Informationen erzeugen und austauschen` umfasst als produktive Komponente vier Aspekte. Der erste Aspekt fokussiert auf das Umwandeln von Informationen. Hierzu gehört es, digitale Informationen zielgerichtet und adressatengerecht aufzubereiten und beispielsweise textbasierte Informationen, Tabellen oder grössere Datensammlungen in visuelle Darstellungsformen wie Diagramme umzuwandeln. Der zweite Aspekt zielt auf das Erzeugen von Informationen ab und bezieht sich auf die Fähigkeit, Informationen oder Informationsprodukte wie ein Poster oder eine Präsentation mit Hilfe computerbasierter Anwendungen für bestimmte Zielsetzungen und Zielgruppen zu erstellen, wobei sowohl inhaltliche als auch layoutbezogene Gestaltungskriterien eine Rolle spielen. Anschaulich gehört hierzu beispielsweise die Erstellung einer digitalen Präsentation zu einem bestimmten Thema oder das Schreiben eines Berichtes, der Informationen aus unterschiedlichen Programmen (z. B. Textverarbeitung, Tabellenkalkulation) anhand unterschiedlicher Repräsentationsformate (z. B. Text, Tabellen, Diagramme) integriert. Der dritte Aspekt dieses zweiten Teilbereiches umfasst Fähigkeiten im Hinblick auf die computergestützte Kommunikation bzw. den Austausch von Informationen. Dazu gehört das Verstehen und Anwenden verschiedener computerbasierter Kommunikationsplattformen wie E-Mail, Wikis, Blogs oder Instant Messaging sowie der Umgang mit sozialen Netzwerken. Dabei spielt die Fähigkeit, für spezifische Kommunikationszwecke das jeweils geeignetste Kommunikationswerkzeug auszuwählen, eine wichtige Rolle. Auf einer reflektierten Ebene gehört dazu auch die Fähigkeit, die Angemessenheit von Informationen in einem bestimmten Kontext zu bewerten sowie die sozialen Auswirkungen geteilter Informationen durch computerbasierte Kommunikationsmedien zu verstehen. Der vierte Aspekt ergänzt den sicheren Umgang mit digitalen Informationen. Dazu 
gehören Kenntnisse über einen reflektierten Umgang mit computerbasierten Informationen sowie ein Verständnis über ethische und rechtliche Grundlagen der Kommunikation und des Austausches digitaler Informationen, auch unter Berücksichtigung von Urheberrechten. Zu ergänzen sei an dieser Stelle, dass die ICILS2013-Datenauswertung und die internationalen Skalierungen eine international sehr hohe latente Korrelation von $r=.96$ zwischen den beiden Teilbereichen aufzeigen (Fraillon et al. 2014). Dieses Ergebnis weist darauf hin, dass sich die theoretisch begründbare Unterscheidung in eine rezeptive und produktive Komponente empirisch nicht abbildet und computer- und informationsbezogene Kompetenzen als eindimensionales Gesamtkonstrukt betrachtet werden können.

\section{Zentrale Ergebnisse aus ICILS 2013 zu computer- und informationsbezogenen Kompetenzen und der Computernutzung in der Schule für Deutschland und die Schweiz}

Um die Kompetenzniveaus der Schülerinnen und Schüler sinnvoll interpretieren zu können, wurde im Rahmen von ICILS 2013 die Metrik der Leistungswerte wie auch in anderen Schulleistungsstudien auf einen Mittelwert von 500 mit einer Standardabweichung von 100 Leistungspunkten transformiert. Das bedeutet, dass der internationale Mittelwert, der sich aus den Ergebnissen aller Schülerinnen und Schüler der teilnehmenden Bildungssysteme zusammensetzt, die die Qualitätsstandards hinsichtlich der Rücklaufquoten erfüllt haben, 500 Punkte beträgt (Eickelmann, Bos, et al. 2014). Die Achtklässlerinnen und Achtklässler in Deutschland erreichen im Schnitt 523 Punkte und Jugendliche in der Schweiz ${ }^{1}$ durchschnittlich 526 Punkte. In beiden Ländern liegen damit die mittleren Kompetenzen der Jugendlichen im Bereich des Mittelwerts der Vergleichsgruppe EU (525 Punkte), welche sich aus allen europäischen Ländern zusammensetzt, die an ICILS 2013 teilgenommen haben und gleichsam EU-Mitglied sind. Weiterhin liegen die mittleren Kompetenzniveaus der Schweizer Stichprobe sowie der Achtklässlerinnen und Achtklässler in Deutschland statistisch signifikant über dem internationalen Mittelwert, aber deutlich unter den mittleren Kompetenzniveaus in der Tschechischen Republik, Kanada (Ontario), Australien, Dänemark, Polen, Norwegen, der Republik Korea und den Niederlanden (Eickelmann, Gerick u. Bos 2014). Weiterhin erreichen in beiden Ländern nur wenige Schülerinnen und Schüler Kompetenzniveaus, die der obersten in ICILS 2013 gebildeten Kompetenzstufe entsprechen (Anteil in der Schweizer Stichprobe: 2,0\%; Deutschland: 1,5\%). Ausserdem sind

1 Im Gegensatz zu Deutschland hat die Schweiz die IEA-Standards für die Schul- und Schüler/innengesamtteilnahmequote nicht erreichen können. Aufgrund des geringeren Rücklaufs sind daher die Ergebnisse für die Schweiz nicht repräsentativ für das gesamte Land, sondern bilden nur die Kompetenz- und Befragungsergebnisse der Achtklässlerinnen und Achtklässler ab, die in der Schweiz an der Studie teilgenommen haben (vgl. Eickelmann, Bos, et al. 2014; Fraillon et al. 2014). 
jeweils die Anteile der Jugendlichen auf den beiden unteren Kompetenzstufen mit 29,8 Prozent für die Schweizer Stichprobe und 29,2 Prozent für Deutschland besorgniserregend hoch (vgl. Bos, Eickelmann u. Gerick 2014). Weiterhin zeigt sich insbesondere in Deutschland, dass Hintergrundmerkmale wie das kulturelle Kapital der Familien bedeutsame Prädiktoren für die computer- und informationsbezogenen Kompetenzen der Jugendlichen darstellen (Wendt et al. 2014). Allerdings ergeben sich für die Schweizer Stichprobe deutlich geringere Disparitäten als in Deutschland (vgl. ebd.). Weiterhin zeigt sich in Deutschland wie in den meisten anderen an ICILS 2013 teilnehmenden Ländern, dass die Mädchen über signifikant höhere Kompetenzen verfügen als die Jungen (Lorenz et al. 2014). Für die in der Schweiz befragten Schülerinnen und Schüler ist der Unterschied nominell ebenfalls sichtbar, aber statistisch nicht signifikant (vgl. ebd.). Dabei ist allerdings zu berücksichtigen, dass die Standardfehler in der Schweizer Stichprobe aufgrund der geringeren Rücklaufquote vergleichsweise hoch sind und daher offen bleibt, ob für eine repräsentative Schweizer Stichprobe ein Kompetenzunterschied zwischen Jungen und Mädchen ebenfalls hätte ausgewiesen werden können.

\section{Häufigkeit der Nutzung neuer Technologien in der Schule sowie insbesondere im Informatikunterricht}

Hinsichtlich der Häufigkeit der Computernutzung im Unterricht lässt sich feststellen, dass diese sowohl aus Lehrer- als auch aus Schüler/innensicht in Deutschland im internationalen Vergleich weit unterdurchschnittlich ist (Eickelmann, Schaumburg, et al. 2014). Lediglich ein Drittel $(34,4 \%)$ der Lehrpersonen in Deutschland nutzt regelmässig (mindestens wöchentlich) Computer im Unterricht, wobei ersichtlich ist, dass jüngere Lehrpersonen den Computer tendenziell häufiger einsetzen. Auch die Schülerinnen und Schüler in Deutschland berichten zu einem vergleichsweise geringen Anteil von nur etwa einem Drittel (31,4\%) von einer regelmässigen Computernutzung in der Schule. Um für die Schülerinnen und Schüler ein gemeinsames Grundverständnis - auch auf internationaler Ebene - zu schaffen, was unter «Computern» zu verstehen ist, wurde in der Einführung in den Fragebögen darüber informiert, dass in der Studie ICILS 2013 unter diesem Begriff sowohl Desktop-Computer, aber auch Notebooks, Laptops, Netbooks und TabletGeräte zu verstehen sind. Hinsichtlich der Häufigkeit der Computernutzung sticht in Deutschland kein Unterrichtsfach positiv hervor und jeweils etwa zwei Drittel der Achtklässlerinnen und Achtklässler geben hier an, in Deutsch, im Fremdsprachenunterricht sowie im Mathematik- und naturwissenschaftlichen Unterricht, nie Computer zu nutzen (vgl. ebd.). Für das Fach Informatik ${ }^{2}$ liegen die Nutzungshäufigkeiten höher, aber sogar hier geben nur 41,7 Prozent der Achtklässlerinnen

2 Zur Bezeichnung des Fachs s. Informationen in der Einleitung. 
und Achtklässler, die das Fach belegen, an, nie Computer im Unterricht zu nutzen. In der Schweizer Stichprobe liegt die Nutzungshäufigkeit von Computern durch Schülerinnen und Schüler in der Schule nur geringfügig über dem Ergebnis für Deutschland (vgl. ebd.). Allerdings ist die Nutzung in den Unterrichtsfächern weiter verbreitet als in Deutschland: Mehr als die Hälfte der Achtklässlerinnen und Achtklässler der Schweizer Stichprobe gibt zumindest für den Sprachenunterricht (Fremd- und Unterrichtssprache) an, mindestens in einigen Stunden Computer zu nutzen. Auch im Informatikunterricht ist hier die Nutzungsquote höher als in Deutschland. Hier geben knapp zwei Drittel $(63,6 \%)$ der befragten Schülerinnen und Schüler an, mindestens in einigen Stunden Computer zu nutzen.

Welchen Beitrag der Informatikunterricht für den Erwerb computer- und informationsbezogener Kompetenzen leistet, ist bisher nicht untersucht. An diese Informations- und Forschungslücke schliesst der vorliegende Beitrag mit den bereits oben skizzierten Forschungsfragen und mit Analysen auf Basis der ICILS-2013-Datengrundlage an.

\section{Forschungsfragen}

Um die Relevanz informatischer Bildung in der Schule für den Erwerb computerund informationsbezogener Kompetenzen für Deutschland und für die Schweiz im internationalen Vergleich zu untersuchen, werden ausgehend von den im Rahmen von Sekundäranalysen gegebenen Möglichkeiten auf der Grundlage der ICILS2013-Datenlage die folgenden Forschungsfragen adressiert:

1. Erreichen Schülerinnen und Schüler der Jahrgangsstufe 8 in Deutschland und in der Schweiz, die das Fach Informatik belegen, höhere computer- und informationsbezogene Kompetenzen, als diejenigen Jugendlichen, die das Fach nicht belegen? Wie stellen sich die Ergebnisse der beiden Länder im internationalen Vergleich dar und welche Rolle spielt dabei, ob im Informatikunterricht Computer überhaupt genutzt werden?

2. Geben Schülerinnen und Schüler, die das Fach Informatik belegen, häufiger an, computerbezogene Fähigkeiten in der Schule erlernt zu haben, als diejenigen, die das Fach nicht belegen? Und welche Rolle spielt diesbezüglich, ob die Achtklässlerinnen und Achtklässler hier Computer nutzen?

3. Geben Schülerinnen und Schüler, die Informatik belegen, ein höheres Mass intrinsischer Motivation und positiver Einstellungen hinsichtlich der Arbeit mit Computern an als solche, die das Fach nicht belegen? Zeigen sich diesbezüglich wiederum Unterschiede hinsichtlich der fachlichen Computernutzung?

4. Wie hängen die in der Schule erlernten computerbezogenen Fähigkeiten sowie die intrinsischen Motive und Einstellungen hinsichtlich der Computernutzung mit den computer- und informationsbezogenen Kompetenzen von 
Schülerinnen und Schülern zusammen, die Informatik belegen und denen, die das Fach nicht belegen? Welche Zusammenhänge ergeben sich hinsichtlich der Computernutzung im Fach Informatik für diejenigen Jugendlichen, die Informatik belegen?

Während die erste Forschungsfrage Kompetenzunterschiede deskriptiv betrachtet und damit den Beitrag des Informatikunterrichts für den Kompetenzerwerb fokussiert, wird mit den nachfolgenden beiden Forschungsfragen darauf hingearbeitet, die unter Forschungsfrage 4 vertiefend betrachteten Kompetenzunterschiede unter Kontrolle von relevanten Faktoren zu untersuchen. Dazu werden in die abschliessenden Zusammenhangsanalysen durch den Einbezug von Hintergrundvariablen wie dem Geschlecht, dem kulturellen Kapitel und der sozialen Lage der Familien (gemessen über die sogenannte Büchervariable) weitere, bereits untersuchte und für das Kompetenzniveau als relevant identifizierte Faktoren (vgl. Bos et al. 2014) einbezogen und hier erstmals mit der Perspektive der Relevanz des Informatikunterrichts unter Kontrolle von Hintergrundvariablen umfassend untersucht.

\section{Die Relevanz von Informatikunterricht für den Erwerb von computer- und informationsbezogenen Kompetenzen in Deutschland und der Schweiz im internationalen Vergleich - Sekundäranalysen auf der Grundlage des inter- nationalen ICILS-2013-Datensatzes}

Die nachfolgenden Analysen werden in der Reihenfolge der vorgenannten Forschungsfragen berichtet. Die Ergebnisse beziehen sich auf die repräsentativ gezogenen Stichproben von Schülerinnen und Schülern der achten Jahrgangsstufe in Deutschland sowie einer in der Schweiz befragten Gruppe (zu Besonderheiten der Stichprobenziehung sowie zu den Rücklaufquoten siehe Eickelmann, Bos, et al. 2014; Fraillon et al. 2014). Zum einen werden in die Analysen die computer- und informationsbezogenen Kompetenzen der Schülerinnen und Schüler der achten Jahrgangsstufe einbezogen, die durch eine computerbasierte Testung ermittelt wurden (vgl. Eickelmann, Bos, et al. 2014). Zum anderen werden Informationen aus dem Hintergrundfragebogen genutzt, den die Jugendlichen am Testtag unmittelbar nach der Kompetenztestung ausgefüllt haben. Diese Hintergrundfragebögen enthalten unter anderem neben Aspekten zum familiären Hintergrund vor allem auch Fragen zur schulischen und fachspezifischen Nutzung von neuen Technologien und hiermit auch für den Informatikunterricht. Hinsichtlich der fachspezifischen Nutzung konnten die Schülerinnen und Schüler in allen beteiligten Bildungssystemen ${ }^{3}$ für verschiedene Fächer auswählen, ob sie nie, in einigen Unterrichtsstunden, in den meisten Unterrichtsstunden oder in jeder bzw. fast jeder Unterrichtsstunde einen Computer im Unterricht nutzen. Jugendliche, die ein Unterrichtsfach

3 Für Norwegen liegen keine Daten zum Informatikunterricht vor. 
nicht belegen, konnten dies ebenfalls kenntlich machen. Es lassen sich demnach für den vorliegenden Beitrag sowohl für die Schweiz als auch für Deutschland die Schülerinnen und Schüler, die Informatik nicht belegen (CH: 19,6\%; DE: 28,6\%), von denen unterscheiden, die das Fach belegen ( $\mathrm{CH}: 80,4 \%$; DE: 71,4\%), wobei die Benennung des Unterrichtsfachs im Zuge der Übersetzung der internationalen Fragebögen an die landesspezifischen Gegebenheiten angepasst wurde (s. o.). Zudem kann innerhalb der Gruppe der Jugendlichen, die angegeben haben, dass sie Informatik belegen, zwischen denjenigen Jugendlichen differenziert werden, die angeben, im Informatikunterricht einen Computer zu nutzen, und denen, die in diesem Fachunterricht nie Computer nutzen.

\section{Kompetenzunterschiede im Bereich der computer- und informationsbezogenen Kompetenzen}

Abbildung 1 zeigt im Hinblick auf die erste Forschungsfrage die Kompetenzunterschiede in den in ICILS 2013 gemessenen computer- und informationsbezogenen Kompetenzen zwischen Achtklässlerinnen und Achtklässlern, die das Fach Informatik belegen und nicht belegen. Abgebildet sind die Ergebnisse für Deutschland sowie die Ergebnisse für die Schweizer Stichprobe im Vergleich zum Mittelwert der an ICILS-2013 teilnehmenden EU-Länder.

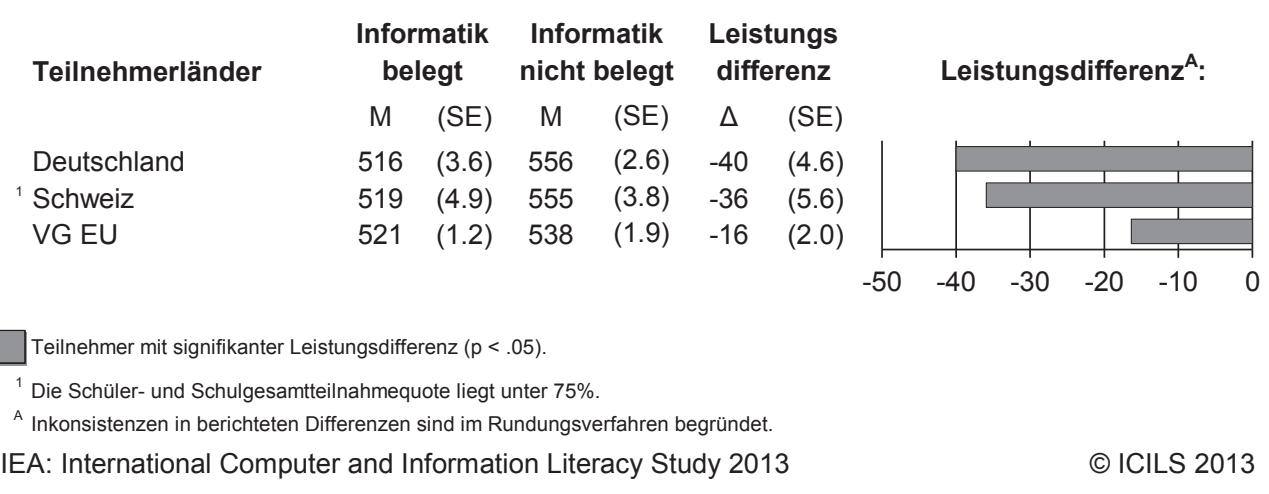

Abb. 1.: Leistungsdifferenzen in den computer- und informationsbezogenen Kompetenzen zwischen Schülerinnen und Schülern, die Informatik belegen, und denen, die das Fach nicht belegen (eigene Darstellung).

Es zeigt sich - möglicherweise überraschend - dass sowohl in Deutschland als auch in der Schweiz die mittleren Kompetenzstände von Jugendlichen, die Informatik nicht belegen, jeweils mit 40 bzw. 36 Leistungspunkten signifikant höher sind als von Schülerinnen und Schülern, die das Fach belegen. Dieser Befund trifft auch für die Vergleichsgruppe VG EU zu (16 Punkte Leistungsdifferenz, siehe Abb. 1). 
Betrachtet man dieses Ergebnis im internationalen Vergleich, so wird deutlich, dass in nur zwei der an ICILS 2013 beteiligten Bildungssysteme, in der Russischen Föderation und in Chile, Schülerinnen und Schüler, die das Fach Informatik oder ein dem Lehrplan der Länder entsprechendes Fach belegen, über signifikant höhere computer- und informationsbezogene Kompetenzen verfügen als ihre gleichaltrigen Mitschülerinnen und Mitschüler (ohne Abbildung). Für dieses Ergebnis gibt es verschiedene mögliche Erklärungsansätze. Neben Unterschieden in den nationalen Curricula und damit der Art und Weise in der in den entsprechenden Lehrplänen der Erwerb computer- und informationsbezogener Kompetenzen, wie sie mit ICILS 2013 konzeptioniert sind, überhaupt angesprochen wird, können auch die Unterschiede im Grad der Verpflichtung des Fachs und damit möglicher Wahloption und dem Wahlverhalten der Schülerinnen und Schüler selbst als Erklärung für die Befundlage dienen. In Deutschland beispielsweise ist in vielen Bundesländern Informatik kein Pflichtfach und wird oftmals im Wahlpflichtbereich neben der dritten Fremdsprache angeboten, die möglicherweise bisher noch eher als Informatik von den leistungsstärkeren Schülerinnen und Schülern gewählt wird. Weiterhin ist inhaltlich anzumerken, dass die Ziele des Informatikunterrichts nicht kongruent mit der Wissensvermittlung im Bereich computer- und informationsbezogener Kompetenzen sind und der Informatikunterricht damit nicht unmittelbar - oder je nach Ausrichtung des Fachs - zumindest nicht ausschliesslich auf den Erwerb von CIL (computer and information literacy) abzielt.

Berichten zudem die Schülerinnen und Schüler, die Informatik belegen, dass sie zumindest in einigen Stunden Computer verwenden, was längst nicht für alle Jugendlichen in der Schweizer Stichprobe und in Deutschland der Fall ist (vgl. Abschnitt 2.3), so verfügen diese Jugendlichen in beiden Ländern über höhere mittlere computer- und informationsbezogene Kompetenzen als diejenigen, die angeben, nie Computer im Informatikunterricht zu nutzen (siehe Abb. 2). 


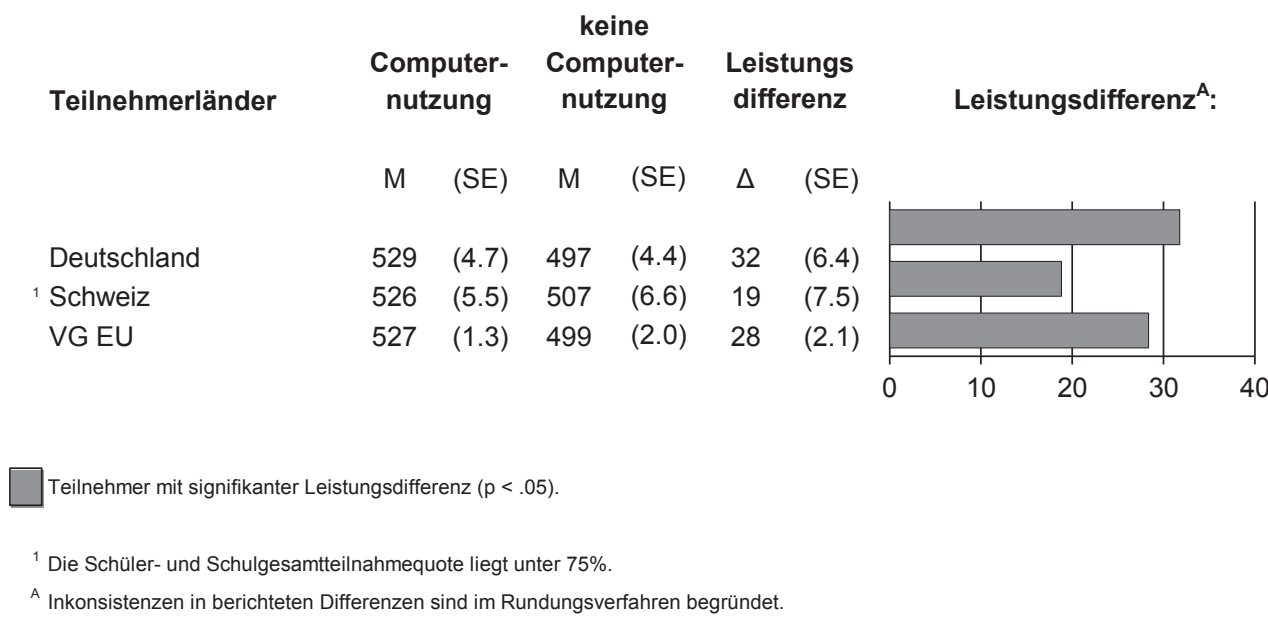

IEA: International Computer and Information Literacy Study 2013

(C) ICILS 2013

Abb. 2.: Differenzen in den computer- und informationsbezogenen Kompetenzen zwischen Schülerinnen und Schülern (Teilstichprobe der Schülerinnen und Schüler, die Informatik belegen), die Computer im Informatikunterricht nutzen und denen, die nie Computer im Informatikunterricht nutzen (eigene Darstellung).

In Deutschland liegt der ermittelte mittlere Kompetenzunterschied bei 32 Leistungspunkten, in der Schweiz beträgt der Unterschied immerhin noch 19 Punkte, jeweils zugunsten der Schülerteilstichprobe, die im Informatikunterricht einen Computer nutzt (siehe Abb. 2). Auch wenn mit ICILS 2013 als Querschnittsstudie keine Kausalitäten geprüft werden können, wirft dieses Ergebnis die Frage nach einer Erklärung dieses Zusammenhangs auf. Neben der einfachen Erklärung, dass die Nutzung von Computern im Informatikunterricht möglicherweise zur Verbesserung der computer- und informationsbezogenen Kompetenzen beiträgt, kann auch begründet werden, dass in Schulen, in denen (mehr) Computer für die unterrichtliche Nutzung - vor allem im Informatikunterricht - zur Verfügung stehen oder die Lehrpersonen fachdidaktisch besser ausgebildet sind und höhere didaktische Kenntnisse zum Einsatz von Computern im Unterricht haben, Schülerinnen und Schüler mit höheren mittleren Leistungsniveaus zu finden sind. Für Deutschland könnte diese Vermutung z. B. für Gymnasien zutreffen, an denen Achtklässlerinnen und Achtklässler im Schnitt ein deutlich höheres mittleres Kompetenzniveau erreichen als Gleichaltrige an nicht-gymnasialen Schulformen (vgl. Bos, Eickelmann u. Gerick 2014). Der Vollständigkeit halber sei auch hier der internationale Vergleich ergänzt: Der betrachtete Leistungsunterschied für Schülerinnen und Schüler, die das Fach Informatik belegen und hier nie Computer nutzen, und denen, die zumindest in einigen Unterrichtsstunden einen Computer nutzen, beträgt in der europäischen Vergleichsgruppe 28 Leistungspunkte und ist international in gleicher 
Weise wie in Deutschland und in der Schweiz in allen teilnehmenden Bildungssystemen ersichtlich und lediglich in Thailand, in der Türkei, in Kanada (Ontario), in Chile sowie in den Niederlanden nicht signifikant (ohne Abbildung). Der grösste mittlere Leistungsvorsprung ist diesbezüglich in Hongkong auszumachen: Hier erreichen die Jugendlichen, die einen Computer im Informatikunterricht nutzen, durchschnittlich 70 Leistungspunkte mehr als die Achtklässlerinnen und Achtklässler, die das Fach Informatik ebenfalls belegen, dort aber nie Computer nutzen (ohne Abbildung). Die Computernutzung scheint also möglicherweise Vorteile mit sich zu bringen. Allerdings erzielt die Gruppe der Jugendlichen, die das Fach Informatik gar nicht belegt, im Mittel auch höhere Kompetenzen als die Teilgruppe der Schülerinnen und Schüler, die Informatik belegt und dort Computer im Unterricht nutzt (siehe Abb. 1 und 2).

\section{Unterschiede in den in der Schule erlernten computerbezogenen Fähigkeiten}

Aus den vorgenannten Erklärungsmustern zu den mittleren Kompetenzunterschieden der Jugendlichen mit und ohne Informatikunterricht sowie der Computernutzung wurde deutlich, dass für eine Erklärung der Kompetenzunterschiede nicht allein die Frage massgeblich sein kann, ob die Schülerinnen und Schüler Informatik als Unterrichtsfach belegen bzw. ob sie dort Computer nutzen oder nicht. Vielmehr scheint es ebenfalls von Bedeutung zu sein, ob Schülerinnen und Schüler sich überhaupt mit dem Erlernen computerbezogener Fähigkeiten befassen. Dies wird in den nachfolgenden Analysen entlang der Forschungsfrage 2 aufgegriffen. In einem ersten Schritt werden dazu die Angaben zur Frage «Hast du in der Schule gelernt, wie die folgenden [computerbezogenen] Aufgaben durchgeführt werden?` für die Achtklässlerinnen und Achtklässler aus Deutschland und für die Schweizer Stichprobe dargestellt. Die Ergebnisse für beide Länder werden wieder differenziert nach Schülerinnen und Schülern, die das Fach Informatik belegen, und solchen, die es nicht belegen, betrachtet und mithilfe des europäischen Vergleichswerts (Mittelwert der Vergleichsgruppe VG EU) verortet (Tab. 1). Ergänzend wird in einem zweiten Schritt - da offensichtlich die Frage nach der unterrichtlichen Computernutzung von hoher Relevanz ist (vgl. Abschnitt 4.1) - geprüft, ob sich wiederum für die Teilstichprobe der Jugendlichen, die das Fach Informatik belegt, abweichende Ergebnisse ergeben. 
Tab. 1.: Angaben zur Frage: «Hast du in der Schule gelernt, wie die folgenden computerbasierten Tätigkeiten durchgeführt werden?», differenziert nach Schülerinnen und Schülern in Deutschland und in der Schweizer Stichprobe, die das Fach Informatik belegt haben, und denen, die das Fach nicht belegt haben (Angaben der Schülerinnen und Schüler in Prozent, dargestellte Kategorie ja; eigene Darstellung).

\begin{tabular}{|c|c|c|c|c|c|c|}
\hline \multirow[b]{2}{*}{ Computerbasierte Tätigkeiten } & \multicolumn{2}{|c|}{$\begin{array}{l}\text { Deutschland } \\
\text { belegt }\end{array}$} & \multicolumn{2}{|c|}{$\begin{array}{r}\text { Schweiz }^{1} \\
\text { belegt }\end{array}$} & \multicolumn{2}{|c|}{ VG EU } \\
\hline & $\begin{array}{c}\text { belegt } \\
\text { Informatik } \\
\%\end{array}$ & $\begin{array}{c}\text { kein } \\
\text { Informatik } \\
\%\end{array}$ & $\begin{array}{c}\text { belegt } \\
\text { Informatik } \\
\%\end{array}$ & $\begin{array}{c}\text { kein } \\
\text { Informatik } \\
\%\end{array}$ & $\begin{array}{c}\text { belegt } \\
\text { Informatik } \\
\%\end{array}$ & $\begin{array}{c}\text { kein } \\
\text { Informatik } \\
\%\end{array}$ \\
\hline Angeben von Internetquellen & 78.4 & 76.7 & 72.5 & 72.1 & 71.6 & 67.7 \\
\hline $\begin{array}{l}\text { Beschaffen von Informationen mithilfe von } \\
\text { Computern }\end{array}$ & 83.3 & 81.4 & 82.8 & 88.2 & 83.6 & 81.1 \\
\hline $\begin{array}{l}\text { Präsentieren von Informationen vor einem } \\
\text { bestimmten Publikum oder zu einem } \\
\text { bestimmten Zweck mit einem Computer }\end{array}$ & 75.8 & 73.5 & 71.3 & 68.7 & 77.6 & 73.3 \\
\hline $\begin{array}{l}\text { Herausfinden, ob Informationen aus dem } \\
\text { Internet vertrauenswürdig sind }\end{array}$ & 48.7 & 37.6 & 49.9 & 46.0 & 66.1 & 63.3 \\
\hline $\begin{array}{l}\text { Entscheiden, welche Informationen für } \\
\text { Hausaufgaben wichtig sind }\end{array}$ & 56.0 & 49.3 & 60.0 & 52.2 & 72.4 & 67.2 \\
\hline $\begin{array}{l}\text { Zusammenstellen von Informationen aus } \\
\text { Internetquellen }\end{array}$ & 72.2 & 69.2 & 63.5 & 59.0 & 68.9 & 64.4 \\
\hline $\begin{array}{l}\text { Entscheiden, wo nach Informationen zu } \\
\text { unbekannten Themen gesucht werden kann }\end{array}$ & 61.8 & 56.5 & 66.3 & 68.7 & 71.2 & 66.6 \\
\hline $\begin{array}{l}\text { Suchen nach unterschiedlichen digitalen } \\
\text { Informationsarten zu einem Thema }\end{array}$ & 55.5 & 45.2 & 66.5 & 62.1 & 67.9 & 63.3 \\
\hline
\end{tabular}

Anmerkung: Fett markiert sind die Prozentzahlen, bei denen sich die beiden Gruppen innerhalb des jeweiligen Bildungssystems signifikant voneinander unterscheiden.

${ }^{1}$ In der Schweiz liegt die Schüler- und Schulgesamtteilnahmequote an ICILS 2013 unter 75\% und die dargestellten Angaben sind daher gemäss IEA-Standards nicht repräsentativ für die Schweiz.

Für die Achtklässlerinnen und Achtklässler in Deutschland zeigt sich, dass Jugendliche, die Informatik belegen, signifikant häufiger angeben, in der Schule gelernt zu haben, ob Informationen aus dem Internet vertrauenswürdig sind (48,7\% vs. $37,6 \%)$, zu entscheiden, welche Informationen für Hausaufgaben wichtig sind (56,0\% vs. $49,3 \%)$, zu entscheiden, wo nach Informationen zu unbekannten Themen gesucht werden kann ( $61,8 \%$ vs. $56,5 \%)$ und nach unterschiedlichen digitalen Informationsarten (gemeint sind z. B. Texte, Bilder und Videos) zu suchen $(55,5 \%$ vs. $45,2 \%)$. In der Tendenz entsprechende Ergebnisse finden sich auch in der Vergleichsgruppe EU (siehe rechte Spalten in Tab. 1). Bei der Interpretation der Ergebnisse ist zu berücksichtigen, dass sich die Angaben auf «in der Schule» beziehen und nicht ausschliesslich auf den Informatikunterricht. Auch vor diesem Hintergrund deckt dieser Befund eine interessante Beobachtung auf: Obwohl in der Zusammenschau die Schülerinnen und Schüler, die Informatik als Fach belegen, wie oben dargestellt, im Mittel über ein geringeres Kompetenzniveau verfügen, geben diese häufiger an, die für den getesteten Bereich relevanten Fähigkeiten in der Schule erlernt zu haben. Dies wirft erneut Fragen hinsichtlich der Wirksamkeit der schulischen Bildung an der Schnittstelle zwischen informatischer Bildung und auf neue Technologien fokussierter Medienbildung auf. Für die Schweizer 
Stichprobe ist zudem anzumerken, dass sich hier kein so deutliches Bild im Sinne von signifikanten Unterschieden zwischen den betrachteten Gruppen zeigt bzw. für ein Item («Beschaffen von Informationen mithilfe von Computern») die Anteile der Achtklässlerinnen und Achtklässler, die angeben, Informatik nicht zu belegen, sogar höher sind, als in der Gruppe der Informatikschülerinnen und -schüler. Weiterhin sei an dieser Stelle ergänzt, dass die schulische Wirksamkeit der Vermittlung von Fertigkeiten im Kontext des Umgangs mit neuen Technologien und digitalen Informationen - wenn überhaupt - sehr spät ansetzt: So konnten Analysen eines in Deutschland durchgeführten nationalen Vertiefungsmoduls zu ICILS 2013 zeigen, dass mit 70,9 Prozent ein erheblicher Anteil der Jugendlichen Autodidakten u. a. im «Finden von Informationen im Internet` sind und sich diese Fertigkeiten selbst beibringen, bevor sie in der Schule thematisiert werden (vgl. Eickelmann, Bos u. Vennemann 2015).

Tab. 2.: Angaben zur Frage: «Hast du in der Schule gelernt, wie die folgenden [computerbezogenen] Aufgaben durchgeführt werden?», differenziert nach Schülerinnen und Schülern (Teilstichprobe der Schülerinnen und Schüler, die Informatik belegt haben), die den Computer in Informatik nutzen, und denen, die keinen Computer im Informatikunterricht nutzen (Angaben der Schülerinnen und Schüler in Prozent, dargestellte Kategorie ja; eigene Darstellung).

\begin{tabular}{|c|c|c|c|c|c|c|}
\hline \multirow[b]{2}{*}{ Computerbasierte Tätigkeiten } & \multicolumn{2}{|c|}{ Deutschland } & \multicolumn{2}{|c|}{ Schweiz $^{1}$} & \multicolumn{2}{|c|}{ VG EU } \\
\hline & $\begin{array}{l}\text { nutzt keinen } \\
\text { Computer in } \\
\text { Informatik } \\
\%\end{array}$ & $\begin{array}{l}\text { nutzt } \\
\text { Computer in } \\
\text { Informatik } \\
\%\end{array}$ & $\begin{array}{l}\text { nutzt keinen } \\
\text { Computer in } \\
\text { Informatik } \\
\% \\
\end{array}$ & $\begin{array}{c}\text { nutzt } \\
\text { Computer in } \\
\text { Informatik } \\
\%\end{array}$ & $\begin{array}{l}\text { nutzt keinen } \\
\text { Computer in } \\
\text { Informatik } \\
\%\end{array}$ & $\begin{array}{c}\text { nutzt } \\
\text { Computer in } \\
\text { Informatik } \\
\%\end{array}$ \\
\hline Angeben von Internetquellen & 75.6 & 80.3 & 66.9 & 75.6 & 63.8 & 73.9 \\
\hline $\begin{array}{l}\text { Beschaffen von Informationen mithilfe von } \\
\text { Computern }\end{array}$ & 81.8 & 84.3 & 77.2 & 86.1 & 75.7 & 85.6 \\
\hline $\begin{array}{l}\text { Präsentieren von Informationen vor einem } \\
\text { bestimmten Publikum oder zu einem } \\
\text { bestimmten Zweck mit einem Computer }\end{array}$ & 69.1 & 80.6 & 65.7 & 74.5 & 68.1 & 80.4 \\
\hline $\begin{array}{l}\text { Herausfinden, ob Informationen aus dem } \\
\text { Internet vertrauenswürdig sind }\end{array}$ & 48.4 & 48.9 & 45.8 & 52.2 & 58.5 & 68.0 \\
\hline $\begin{array}{l}\text { Entscheiden, welche Informationen für } \\
\text { Hausaufgaben wichtig sind }\end{array}$ & 57.5 & 54.9 & 53.1 & 63.9 & 65.3 & 74.3 \\
\hline $\begin{array}{l}\text { Zusammenstellen von Informationen aus } \\
\text { Internetquellen }\end{array}$ & 69.1 & 74.3 & 57.1 & 67.1 & 60.8 & 71.3 \\
\hline $\begin{array}{l}\text { Entscheiden, wo nach Informationen zu } \\
\text { unbekannten Themen gesucht werden kann }\end{array}$ & 60.0 & 63.0 & 57.4 & 71.5 & 64.2 & 73.1 \\
\hline $\begin{array}{l}\text { Suchen nach unterschiedlichen digitalen } \\
\text { Informationsarten zu einem Thema }\end{array}$ & 54.8 & 56.0 & 60.7 & 69.8 & 59.9 & 70.2 \\
\hline
\end{tabular}

Anmerkung: Fett markiert sind die Prozentzahlen, bei denen sich die beiden Gruppen innerhalb des jeweiligen Bildungssystems signifikant voneinander unterscheiden.

${ }^{1}$ In der Schweiz liegt die Schüler- und Schulgesamtteilnahmequote an ICILS 2013 unter 75\% und die dargestellten Angaben sind daher gemäss IEA-Standards nicht repräsentativ für die Schweiz.

Fokussiert man die in diesem Abschnitt durchgeführten Analysen wiederum nur auf diejenigen Schülerinnen und Schüler, die Informatik als Fach belegen, und differenziert hier erneut zwischen denjenigen, die angeben mindestens in einigen Stunden im Informatikunterricht einen Computer zu nutzen, und denjenigen, die 
angeben, nie einen Computer in Informatik zu nutzen, so zeigt sich insbesondere für die Schweizer Stichprobe, dass die Anteile in der Gruppe der Computernutzer statistisch signifikant grösser sind (siehe Tab. 2). Dieses recht deutliche Ergebnis lässt sich über fast alle an ICILS 2013 teilnehmenden Länder hinweg festmachen. Demgegenüber lassen sich entsprechende Unterschiede in Deutschland in dieser Deutlichkeit nicht ausmachen. Lediglich in Bezug auf das computerbasierte Präsentieren von Informationen gibt hier ein höherer Anteil an Informatikschülerinnen und -schülern, die zudem Computer im Informatikunterricht nutzen, an, dies in der Schule thematisiert zu haben $(80,6 \%$ vs. $69,1 \%)$.

\section{Unterschiede hinsichtlich der intrinsischen Motivation und der Einstellung der Schülerinnen und Schüler im Umgang mit neuen Technologien und digitalen Informationen}

Ausgehend von der Annahme, dass motivationale Effekte und Einstellungen ebenfalls einen Zusammenhang zu computer- und informationsbezogenen Kompetenzen haben und sich hier Unterschiede zwischen Schülerinnen und Schülern, die am Informatikunterricht oder einem ähnlich zu verortenden unterrichtlichen Fach oder Teilbereich eines Faches teilnehmen, begründbar wären, werden im nachfolgenden Abschnitt vertiefend Aspekte der Motivation und der Einstellungen betrachtet. Konkret geht es um Fragen zu allgemeinen Motiven der Computernutzung und Einstellungen zu Computern, die über die Zustimmung zu Aussagen wie «Es ist wichtig für mich, mit Computern zu arbeiten», «lch finde, dass Computernutzung Spass macht» oder «Es gefällt mir, neue Dinge am Computer zu lernen» erhoben wurden. Für die nachfolgenden Analysen werden die vier für diese Items erhobenen Antwortkategorien dichotomisiert und dazu die Kategorien trifft zu und trifft eher zu (in trifft zu) zusammengefasst.

Es zeigt sich, dass etwa 90 Prozent der Jugendlichen in Deutschland, in der Schweiz sowie in der Vergleichsgruppe EU unabhängig davon, ob sie Informatik belegen oder nicht, es für sich als wichtig einschätzen, mit Computern zu arbeiten und Gefallen daran finden, neue Dinge am Computer zu erlernen. Auch die Aussage «lch finde, dass Computernutzung Spass macht», erhält unabhängig von den beiden betrachteten Gruppen von über 90 Prozent der Jugendlichen Zustimmung (Tab. 3). Darüber hinaus zeigt sich, dass sich auch keine Unterschiede zwischen den betrachteten Gruppen hinsichtlich der Einschätzung darüber ergeben, dass es Spass macht, Arbeit mit einem Computer zu erledigen (80\% Zustimmung). Die Analysen ergeben weiterhin, dass sich die Gruppen sowohl in Deutschland als auch in der Schweizer Stichprobe nur hinsichtlich des Items «Ich benutze Computer, weil mich die Technik sehr interessiert» unterscheiden und sich hier Unterschiede zwischen Schülerinnen und Schülern abbilden, die Informatik als Unterrichtsfach belegen, 
und denjenigen, die kein Informatik belegen. Während jeweils mehr als die Hälfte (DE: 53,6\% und CH: 51,4\%) der befragten Jugendlichen, die Informatik belegen, sowohl in der Schweizer Stichprobe als auch in Deutschland angeben, dass sie Computer nutzen, weil sie sich für Technik interessieren, ist der Anteil unter den Nicht-Informatikschülerinnen und -schülern mit nur etwas mehr als zwei Fünfteln (DE: 42,4\% und $\mathrm{CH}: 40,4 \%$ ) deutlich geringer. Dieser Unterschied ist auch in der VG EU erkennbar (siehe Tab. 3) und findet sich so auch in den meisten anderen an ICILS 2013 teilnehmenden Bildungssystemen (ohne Abbildung). Dies könnte inhaltlich darauf hindeuten, dass Informatik häufiger von Schülerinnen und Schülern gewählt wird, die sich für Technik interessieren. In Deutschland zeigen sich zudem zwei weitere Unterschiede zwischen den betrachteten Gruppen. Ein signifikant höherer Anteil der Schülerinnen und Schüler, die Informatik belegen, sucht oft nach neuen Möglichkeiten, Dinge mit dem Computer zu erledigen (65,7\% vs. $55,9 \%)$. Im Gegensatz dazu nutzt ein kleiner, jedoch signifikant höherer Anteil der Jugendlichen, die keinen Informatikunterricht besuchen, das Internet gerne, um Informationen zu suchen (91,2\% vs. $88,1 \%)$.

Tab. 3.: Motive und Einstellungen zur Computernutzung, differenziert nach Schülerinnen und Schülern, die das Fach Informatik belegen, und denen, die das Fach nicht belegen (Angaben der Schülerinnen und Schüler in Prozent, Kategorie trifft zu; eigene Darstellung).

\begin{tabular}{|c|c|c|c|c|c|c|}
\hline \multirow[b]{2}{*}{$\begin{array}{l}\text { Motive und Einstellungen } \\
\text { der Computernutzung }\end{array}$} & & \multicolumn{2}{|c|}{ Schweiz $^{1}$} & \multicolumn{2}{|c|}{ VG EU } \\
\hline & $\begin{array}{c}\text { belegt } \\
\text { Informatik } \\
\%\end{array}$ & $\begin{array}{c}\text { belegt } \\
\text { kein } \\
\text { Informatik } \\
\%\end{array}$ & $\begin{array}{c}\text { belegt } \\
\text { Informatik } \\
\%\end{array}$ & $\begin{array}{c}\text { belegt } \\
\text { kein } \\
\text { Informatik } \\
\%\end{array}$ & $\begin{array}{c}\text { belegt } \\
\text { Informatik } \\
\%\end{array}$ & $\begin{array}{c}\text { belegt } \\
\text { kein } \\
\text { Informatik } \\
\%\end{array}$ \\
\hline $\begin{array}{l}\text { Es ist sehr wichtig für mich, mit Computern } \\
\text { zu arbeiten }\end{array}$ & 89.9 & 87.2 & 84.4 & 87.4 & 91.6 & 92.0 \\
\hline $\begin{array}{l}\text { Ich finde, dass Computernutzung Spass } \\
\text { macht }\end{array}$ & 96.1 & 96.6 & 90.9 & 92.1 & 94.8 & 95.7 \\
\hline $\begin{array}{l}\text { Es macht mir mehr Spass, meine Arbeit mit } \\
\text { einem Computer zu erledigen als ohne }\end{array}$ & 81.3 & 77.0 & 76.8 & 74.2 & 83.1 & 83.7 \\
\hline $\begin{array}{l}\text { Ich benutze Computer, weil mich die } \\
\text { Technik sehr interessiert }\end{array}$ & 53.6 & 42.4 & 51.4 & 40.4 & 57.7 & 52.9 \\
\hline $\begin{array}{l}\text { Es gefällt mir, neue Dinge am Computer zu } \\
\text { lernen }\end{array}$ & 89.3 & 87.8 & 83.2 & 84.3 & 89.9 & 89.2 \\
\hline $\begin{array}{l}\text { Ich suche oft nach neuen Möglichkeiten, } \\
\text { Dinge mit dem Computer zu erledigen }\end{array}$ & 65.7 & 55.9 & 65.6 & 64.4 & 73.4 & 71.4 \\
\hline $\begin{array}{l}\text { Ich nutze das Internet gern, um neue } \\
\text { Informationen zu suchen }\end{array}$ & 88.1 & 91.2 & 88.1 & 91.0 & 89.8 & 89.9 \\
\hline
\end{tabular}

Anmerkung: Fett markiert sind die Prozentzahlen, bei denen sich die beiden Gruppen innerhalb des jeweiligen

Bildungssystems signifikant voneinander unterscheiden.

${ }^{1}$ In der Schweiz liegt die Schüler- und Schulgesamtteilnahmequote an ICILS 2013 unter 75\% und die dargestellten Angaben sind daher gemäß IEA-Standards nicht repräsentativ für die Schweiz.

Warum sich insbesondere für Deutschland und die befragten Schweizer Jugendlichen keine weiteren Unterschiede finden lassen, muss für jedes der beiden Bildungssysteme auch unter Berücksichtigung der spezifischen Rahmenbedingungen getrennt untersucht werden. Dabei ist der Frage nachzugehen, warum für 
die informatische Bildung in der Schule zumindest aus Sicht der Schülerinnen und Schüler der technische Aspekt solch einen auffälligen Zusammenhang zeigt. Weiterhin ist für Interpretationen zu beachten, inwieweit die Belegung des Faches Informatik verbindlich ist und im Falle einer verpflichtenden Verankerung ohnehin nur noch im eingeschränkten Masse die Motivlagen und die Einstellungen zu berücksichtigen sind. Hier käme zum Tragen, dass in diesem Fall alle Schülerinnen und Schüler das Fach unabhängig von ihrer persönlichen Interessens- und Motivationslage belegen müssen.

Zu ergänzen ist, dass es in beiden in diesem Beitrag betrachteten Ländern, also sowohl in Deutschland als auch in der Schweiz, Unterschiede innerhalb der Gruppe der Jugendlichen, die Informatik belegt, gibt und auch hier die Nutzung des Computers bzw. die Grundfrage, ob Computer im Informatikunterricht genutzt werden, zumindest für einige der betrachteten Items zu Motiven und Einstellungen gegenüber Computertechnologien ausschlaggebend zu sein scheint (siehe Tab. 4).

Tab. 4.: Motive und Einstellungen zur Computernutzung, Teilstichprobe der Schülerinnen und Schüler, die Informatik belegt haben, differenziert nach Jugendlichen, die den Computer im Informatikunterricht nutzen, und denen, die nie Computer im Informatikunterricht nutzen (Angaben der Schülerinnen und Schüler in Prozent, Kategorie trifft zu; eigene Darstellung).

\begin{tabular}{|c|c|c|c|c|c|c|}
\hline $\begin{array}{l}\text { Motive und Einstellungen } \\
\text { der Computernutzung }\end{array}$ & $\begin{array}{l}\text { Deutsc } \\
\text { nutzt keinen } \\
\text { Computer in } \\
\text { Informatik } \\
\% \\
\end{array}$ & $\begin{array}{l}\text { chland } \\
\text { nutzt } \\
\text { Computer in } \\
\text { Informatik } \\
\%\end{array}$ & $\begin{array}{c}\text { Schw } \\
\text { nutzt keinen } \\
\text { Computer in } \\
\text { Informatik } \\
\% \\
\end{array}$ & $\begin{array}{l}\text { veiz }^{1} \\
\text { nutzt } \\
\text { Computer in } \\
\text { Informatik } \\
\%\end{array}$ & $\begin{array}{l}\text { VG } \\
\text { nutzt keinen } \\
\text { Computer in } \\
\text { Informatik } \\
\%\end{array}$ & $\begin{array}{l}\text { EU } \\
\text { nutzt } \\
\text { Computer in } \\
\text { Informatik } \\
\% \\
\end{array}$ \\
\hline $\begin{array}{l}\text { Es ist sehr wichtig für mich, mit Computern } \\
\text { zu arbeiten }\end{array}$ & 89.0 & 90.7 & 81.2 & 86.2 & 89.4 & 92.3 \\
\hline $\begin{array}{l}\text { Ich finde, dass Computernutzung Spass } \\
\text { macht }\end{array}$ & 94.5 & 97.3 & 87.3 & 92.9 & 92.6 & 95.3 \\
\hline $\begin{array}{l}\text { Es macht mir mehr Spass, meine Arbeit mit } \\
\text { einem Computer zu erledigen als ohne }\end{array}$ & 79.9 & 82.3 & 72.7 & 79.2 & 80.5 & 83.8 \\
\hline $\begin{array}{l}\text { Ich benutze Computer, weil mich die } \\
\text { Technik sehr interessiert }\end{array}$ & 51.6 & 55.1 & 46.3 & 54.3 & 56.0 & 59.5 \\
\hline $\begin{array}{l}\text { Es gefällt mir, neue Dinge am Computer zu } \\
\text { lernen }\end{array}$ & 87.5 & 90.6 & 75.0 & 87.9 & 86.1 & 91.2 \\
\hline $\begin{array}{l}\text { Ich suche oft nach neuen Möglichkeiten, } \\
\text { Dinge mit dem Computer zu erledigen }\end{array}$ & 64.9 & 66.2 & 60.9 & 68.4 & 68.6 & 75.1 \\
\hline $\begin{array}{l}\text { Ich nutze das Internet gern, um neue } \\
\text { Informationen zu suchen }\end{array}$ & 85.1 & 90.1 & 82.5 & 91.3 & 86.4 & 90.7 \\
\hline
\end{tabular}

Informationen zu suchen $\quad 85.1 \quad 86.5$

Bildungssystems signifikant voneinander unterscheiden.

${ }^{1}$ In der Schweiz liegt die Schüler- und Schulgesamtteilnahmequote an ICILS 2013 unter 75\% und die dargestellten Angaben sind daher gemäß IEA-Standards nicht repräsentativ für die Schweiz.

Werden Computer im Informatikunterricht genutzt, so geben beispielsweise sowohl in Deutschland, in der Schweizer Stichprobe als auch in der Vergleichsgruppe EU signifikant mehr Achtklässlerinnen und Achtklässler an, dass die Computernutzung ihnen Spass macht (DE: 97,3\% vs. 94,5\%, CH: $92,9 \%$ vs. $87,3 \%$ und VG EU 
$95,3 \%$ vs. $92,6 \%)$ und dass sie das Internet gerne nutzen, um neue Informationen zu suchen (DE: $90,1 \%$ vs. $85,1 \%$, CH: $91,3 \%$ vs. $82,5 \%$ und VG EU $90,7 \%$ vs. $86,4 \%$ ) als die Jugendlichen, die keinen Computer nutzen. In der Schweizer Stichprobe ist zudem auch der Anteil bezüglich der Aussagen darüber, ob Computer genutzt werden, weil sich die Jugendlichen für Technik interessieren und dass es ihnen gefällt, neue Dinge am Computer zu lernen, grösser als bei den Computernutzern. Auch international findet sich diese Tendenz sehr deutlich und es zeigt sich, dass Jugendliche, die Computer im Informatikunterricht nutzen, eine positivere Grundhaltung zu den verschiedenen genannten Aspekten aufweisen. Am deutlichsten zeigt sich dieses Bild in der Republik Korea, wo die Unterschiede für alle sieben abgefragten Aspekte signifikant zugunsten der Jugendlichen, die Computer im Informatikunterricht nutzen, sind.

\section{Haben die gefundenen Kompetenzunterschiede Bestand? - Zusammenhangs- analysen unter Kontrolle von Hintergrundmerkmalen}

Im Folgenden wird getrennt für Deutschland und für die Schweizer Stichprobe schrittweise regressionsanalytisch untersucht (vgl. zur Methode u. a. Cohen et al. 2013), wie die in der Schule erlernten computerbezogenen Fähigkeiten sowie die intrinsischen Motive und Einstellungen hinsichtlich der Computernutzung mit den computer- und informationsbezogenen Kompetenzen von Schülerinnen und Schülern zusammenhängen, die Informatik belegen und denen, die das Fach nicht belegen. In der Regression werden dazu die Schülerinnen und Schüler, die angeben, Informatik nicht zu belegen, als Referenzgruppe betrachtet. Zudem werden als Hintergrundmerkmale die Anzahl der Bücher zu Hause als Indikator für das kulturelle Kapital und die soziale Lage der Familien, sowie das Geschlecht der Jugendlichen in die Regression einbezogen. Die unter Abschnitt 2 und 3 betrachteten Items zu den in der Schule erlernten computerbezogenen Fähigkeiten sowie die Fragen zu intrinsischen Motiven und Einstellungen zur Computernutzung werden jeweils zu Indizes zusammengefasst in die Regression aufgenommen. Diese Indizes wurden bereits im Rahmen der internationalen Berichtlegung der Studie gebildet (vgl. Fraillon et al. 2014) und auf einen Mittelwert von 50 sowie eine Standardabweichung von 10 transformiert. In den ersten internationalen Index (Cronbachs Alpha: .81; Fraillon et al. 2015) zu den in der Schule erlernten computerbezogenen Fähigkeiten der Jugendlichen gehen insgesamt acht Items ein (siehe Tab. 1 und 2). Der zweite betrachtete internationale Index zu den Motiven und Einstellungen der Computernutzung (Cronbachs Alpha: .81; ebd.) wird aus sieben Items aus dem Fragebogen gebildet (siehe Tab. 3 und 4). 
Tab. 5.: Regressionsmodell zur Erklärung der computer- und informationsbezogenen Kompetenzen der Schülerinnen und Schüler in Deutschland (eigene Darstellung).

\begin{tabular}{|c|c|c|c|c|c|c|}
\hline & \multicolumn{2}{|c|}{ Modell I } & \multicolumn{2}{|c|}{ Modell II } & \multicolumn{2}{|c|}{ Modell III } \\
\hline & $\mathrm{b}$ & $(\mathrm{SE})$ & $\mathrm{b}$ & $(\mathrm{SE})$ & $\mathrm{b}$ & (SE) \\
\hline \multicolumn{7}{|l|}{ Informatik Belegung } \\
\hline Informatik belegt + keine Computernutzung ${ }^{A}$ & -47.9 & $(6.8)$ & -47.6 & (6.9) & -44.1 & (6.7) \\
\hline Informatik belegt + Computernutzung ${ }^{\mathrm{A}}$ & -29.1 & (6.4) & -29.0 & (6.3) & -26.2 & (6.3) \\
\hline \multicolumn{7}{|c|}{ Schulisch erlernte Fähigkeiten und Motivation/Einstellungen } \\
\hline Schulisch erlernte Tätigkeiten ${ }^{B}$ & - & - & -0.2 & 0.2 & -0.2 & 0.2 \\
\hline Motivation der Compuzernuternutzung ${ }^{\mathrm{B}}$ & - & - & 0.1 & 0.2 & 0.4 & 0.2 \\
\hline \multicolumn{7}{|l|}{ Hintergrundvariablen } \\
\hline Buchbesitz im Haushalt $^{\mathrm{C}}$ & - & - & - & - & 35.4 & 3.7 \\
\hline Geschlecht $^{\mathrm{D}}$ & - & - & - & - & 17.7 & 3.8 \\
\hline Konstante & \multicolumn{2}{|c|}{555} & \multicolumn{2}{|c|}{548} & \multicolumn{2}{|c|}{530} \\
\hline $\mathrm{R}^{2}$ & \multicolumn{2}{|c|}{0.09} & \multicolumn{2}{|c|}{0.09} & \multicolumn{2}{|c|}{0.15} \\
\hline
\end{tabular}

Anmerkungen:

$\mathrm{b}=$ Regressionsgewichte (unstandardisiert)

Abhängige Variable: Computer- und Informationsbezogene Kompetenzen

Signifikante Koeffizienten $(p<.05)$ sind fett gedruckt.

${ }^{A}$ Referenzgruppe: Kein Informatik belegt

${ }^{B}$ Skalierter und international auf $\mathrm{M}=50$ und $\mathrm{SD}=10$ transformierter Index

C 0 - Maximal 200 Bücher; 1 - Mehr als 200 Bücher

${ }^{\mathrm{D}} 0$ - Junge; 1 - Mädchen

Im Ergebnis zeigt sich für Deutschland und auch für die Schweizer Stichprobe (Tab. 5 und 6) im ersten Regressionsschritt, dass sowohl die Jugendlichen, die Informatik belegen und keinen Computer nutzen, als auch die Schülerinnen und Schüler, die Informatik belegen und einen Computer im Informatikunterricht nutzen, geringere mittlere computer- und informationsbezogene Kompetenzen aufweisen als die Achtklässlerinnen und Achtklässler, die das Fach Informatik nicht belegen (Modell I). Dabei weist die Gruppe, die im Informatikunterricht keinen Computer nutzt, deutlich geringere mittlere Kompetenzen auf als die Jugendlichen mit Computernutzung im Informatikunterricht. Dies bildet inhaltlich die Ergebnisse der deskriptiven Analysen zu Forschungsfrage 1 erneut ab (Abschnitt 4.1). Das Modell weist eine Varianzaufklärung von 9 Prozent in Deutschland und 5 Prozent in der Schweizer Stichprobe auf. In den nachfolgenden erweiterten Regressionsmodellen zeigt sich, dass die vorgenannten Ergebnisse des ersten Regressionsschritts in beiden Ländern auch unter Kontrolle der schulisch erlernten computerbezogenen Fähigkeiten und der Motive bzw. Einstellungen zur Computernutzung (Modell II) sowie zusätzlich unter Berücksichtigung der Hintergrundmerkmale (Modell III) bestehen. Hinsichtlich der schulisch erlernten computerbezogenen Fähigkeiten und den Motiven bzw. Einstellungen der Computernutzung ist weiterhin ersichtlich, dass lediglich in Deutschland die Motive bzw. Einstellungen zur Computernutzung im Gesamtmodell einen signifikanten, wenn auch kleinen Prädiktor der computer- und informationsbezogenen Kompetenzen darstellen ( $b=0.4$; unstandardisiertes Regressionsgewicht, das wie Leistungspunkte interpretiert werden 
kann). Ein weiterer Unterschied zwischen den beiden betrachteten Ländern zeigt sich in Bezug auf die Hintergrundmerkmale: Während in Deutschland sowohl der Buchbesitz im Haushalt $(b=35.4)$ als auch das Geschlecht (zugunsten der Mädchen mit $b=17.7$ ) bedeutsame Prädiktoren der computer- und informationsbezogenen Kompetenzen darstellen, ist in der Schweizer Stichprobe lediglich der Buchbesitz als Indikator für das kulturelle Kapital sowie die soziale Lage der Familien ein signifikanter Faktor ( $b=22.8)$, was dem Ergebnis der Analysen für die gesamte Schweizer Stichprobe entspricht (vgl. Abschnitt 2.3 und Lorenz et al. 2014). Zu ergänzen ist, dass das Gesamtmodell (III) in Deutschland 15 Prozent der Varianz aufklärt. In der Schweizer Stichprobe sind es lediglich 7 Prozent.

Tab. 6.: Regressionsmodell zur Erklärung der computer- und informationsbezogenen Kompetenzen der Schülerinnen und Schüler in der Schweizer Stichprobe ${ }^{1}$ (eigene Darstellung).

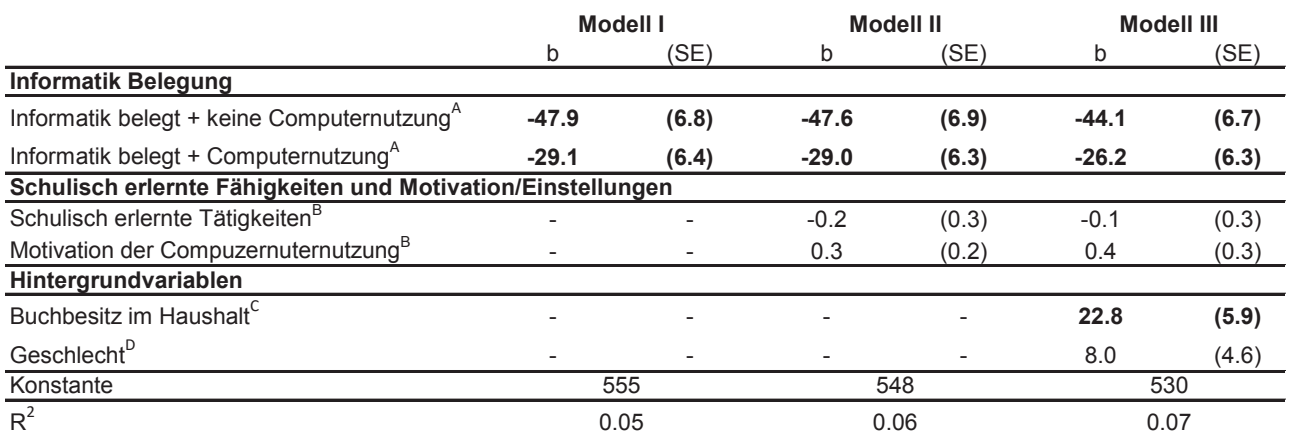

Anmerkungen:

$\mathrm{b}=$ Regressionsgewichte (unstandardisiert)

Abhängige Variable: Computer- und Informationsbezogene Kompetenzen

Signifikante Koeffizienten $(p<.05)$ sind fett gedruckt.

${ }^{A}$ Referenzgruppe: Kein Informatik belegt

${ }^{B}$ Skalierter und international auf $\mathrm{M}=50$ und $\mathrm{SD}=10$ transformierter Index

${ }^{C} 0$ - Maximal 200 Bücher; 1 - Mehr als 200 Bücher

${ }^{D} 0$ - Junge; 1 - Mädchen

${ }^{1}$ In der Schweiz liegt die Schüler- und Schulteilnahmequote an ICILS 2013 unter $75 \%$ und die dargestellten Angaben sind daher gemäß IEA-Standards nicht repräsentativ für die Schweiz.

\section{Zusammenfassung und Diskussion der Ergebnisse und Ausblick}

Vor dem Hintergrund, dass mit ICILS 2013 erstmalig international vergleichend computer- und informationsbezogene Kompetenzen von Jugendlichen im achten Schuljahr computerbasiert mit repräsentativ gezogenen Stichproben untersucht wurden und sowohl die Jugendlichen in Deutschland als auch in der Schweiz im Mittel im internationalen Vergleich eher mittelmässig abgeschnitten haben, stellt sich für die beiden Bildungssysteme die Frage, in welchen Fächern die betrachteten fächerübergreifenden Schlüsselkompetenzen in der Schule besonders gut gefördert werden können. In diesem Zusammenhang wird in der aktuellen Diskussion 
auch der informatischen Bildung und damit insbesondere dem Fach Informatik eine besondere Bedeutung zugeschrieben, da dieser Bereich zumindest ansatzweise eine im Vergleich zu anderen Fächergruppen höhere theoretisch-konzeptionelle Affinität zum Bereich des kompetenten Umgangs mit neuen Technologien und digitalen Informationen aufweist.

Vor diesem Hintergrund wurde in diesem Beitrag mittels Sekundäranalysen der ICILS-2013-Stichprobe untersucht, inwieweit sich in Deutschland und in der Schweiz die computer- und informationsbezogenen Kompetenzen von Schülerinnen und Schülern, die das Fach Informatik belegen, von den Kompetenzständen der Jugendlichen unterscheiden, die das Fach nach eigenen Angaben nicht belegen. Im Ergebnis zeigt sich - anders als zunächst zu vermuten gewesen wäre - dass in allen an ICILS 2013 teilnehmenden Bildungssystemen, demnach auch in Deutschland und in der Schweiz, diejenigen Schülerinnen und Schüler im Mittel signifikant höhere computer- und informationsbezogene Kompetenzen aufweisen, die das Fach Informatik nicht belegen. Da sich in der ersten Berichtslegung der Studie ICILS 2013 bereits gezeigt hatte, dass längst nicht alle Schülerinnen und Schüler, die in Deutschland das Fach Informatik belegen, auch einen Computer im Informatikunterricht nutzen (Eickelmann, Schaumburg, et al. 2014, vgl. für die Schweiz auch Abschnitt 2.3), was für den Erwerb der computer- und informationsbezogenen Kompetenzen allerdings möglicherweise bedeutsam scheint, wurde zudem in diesem Beitrag untersucht, ob sich innerhalb der Teilstichprobe der Jugendlichen, die Informatik belegen, Unterschiede zwischen den Schülerinnen und Schülern ergeben, die dort einen Computer nutzen, und denen, die keinen Computer im Informatikunterricht nutzen. Die Analysen zeigen, dass die Jugendlichen, die einen Computer in Informatik nutzen, im Mittel signifikant höhere computerund informationsbezogene Kompetenzen erzielen als diejenigen Jugendlichen, die keinen Computer im Informatikunterricht nutzen. Anzumerken ist hier, dass die mittleren computer- und informationsbezogenen Kompetenzen der Schülerinnen und Schüler, die keinen Informatikunterricht belegen, im Vergleich allerdings immer noch höher ausfallen. In der Interpretation dieser Ergebnisse stellt sich die Frage, wie zu begründen ist, dass Schülerinnen und Schüler, die Informatik belegen, im Mittel geringere computer- und informationsbezogene Kompetenzen aufweisen als die Jugendlichen, die das Fach nicht belegen. Hier erweisen sich möglicherweise zwei Begründungslinien als entscheidend: Zum einen wäre es im Anschluss an die vorliegenden Analysen zielführend, genauer zu untersuchen, inwieweit sich tatsächlich Überschneidungen zwischen dem in ICILS 2013 gemessenen Konstrukt der computer- und informationsbezogenen Kompetenzen (vgl. dazu u. a. Abschnitt 2.1) und den nationalen Curricula der Bildungssysteme im Bereich der informatischen Bildung ergeben und hier Zusammenhänge tatsächlich begründet wären. Zum anderen, und dies ist vielleicht der entscheidendere Aspekt, 
scheint es lohnenswert, die Gruppe, die das Fach Informatik belegt, genauer zu analysieren. Daran anknüpfend wäre für Deutschland zu berücksichtigen, dass Informatik in vielen Bundesländern kein Pflichtfach ist und dies möglicherwiese im Wahlpflichtbereich bisher eher von leistungsschwächeren Schülerinnen und Schülern gewählt wird. Zudem könnte es sein, dass Informatik vermehrt von männlichen Jugendlichen gewählt wird, die in keinem an ICILS 2013 beteiligten Bildungssystem im Mittel höhere computer- und informationsbezogene Kompetenzen erzielt haben als ihre weiblichen Mitschülerinnen (vgl. Lorenz et al. 2014). Auch diesem Erklärungsansatz wäre mit weiteren Forschungsarbeiten nachzugehen. Da es auch von zentraler Bedeutung scheint, ob Schülerinnen und Schüler sich überhaupt mit dem Erlernen computerbezogener Fähigkeiten befassen, wurde im vorliegenden Beitrag in einem weiteren Schritt untersucht, inwieweit sich die Jugendlichen, die Informatik belegen, von denen, die das Fach nicht belegen, hinsichtlich ihrer Einschätzung unterscheiden, ob sie verschiedene computerbezogene Fähigkeiten in der Schule erworben haben. Obwohl die Schülerinnen und Schüler, die Informatik als Fach belegen, im Mittel über ein geringeres Kompetenzniveau verfügen, geben sie zumindest in Deutschland und auch in der in ICILS 2013 gebildeten Vergleichsgruppe VG EU häufiger an, die für den getesteten Bereich relevanten Fähigkeiten in der Schule erlernt zu haben und hier vor allem die Jugendlichen, die im Informatikunterricht einen Computer nutzen. Dies wirft erneut die Frage nach der Wirksamkeit der schulischen Bildung an der Schnittstelle zwischen informatischer Bildung und einer auf neue Technologien fokussierten Medienbildung auf. Zudem wurden in diesem Beitrag die Unterschiede hinsichtlich der intrinsischen Motivation und der Einstellung der Schülerinnen und Schüler im Umgang mit neuen Technologien und digitalen Informationen differenziert nach Jugendlichen, die Informatik belegen, und denjenigen, die das Fach nicht belegen, betrachtet. Auffällig ist hier der Befund, dass Informatik häufiger von Jugendlichen belegt wird, die sich für Technik interessieren. Alle berichteten Befunde hinsichtlich der mittleren Leistungsunterschiede in den computer- und informationsbezogenen Kompetenzen bleiben auch bestehen, wenn man die Angaben der Jugendlichen hinsichtlich der in der Schule erlernten computerbezogenen Fähigkeiten sowie die Motivation und Einstellungen zur Computernutzung hinzuzieht, ebenso wie das Geschlecht der Jugendlichen und das kulturelle Kapital der Familien.

Im schulischen Kontext wird es zukünftig stärker als bisher die Aufgabe aller Fächer sein, einen zielgerichteten und reflektierten Umgang mit neuen Technologien und digitalen Informationen zu unterstützen und Medienbildung voranzutreiben und fachintegrativ zu verankern. Das Fach Informatik bzw. informatische Bildung insgesamt kann - je nach inhaltlicher Ausgestaltung - diesbezüglich einen spezifischen Beitrag leisten. Für diesen Bereich ergibt sich daraus zukünftig ein besonderer Bildungsauftrag, der hier informatische Bildung einerseits stärker in die 
Pflicht nimmt und andererseits durch seine Affinität zu fächerübergreifenden Kompetenzbereichen im Hinblick auf Medienbildung mit steigender Relevanz auch neue Möglichkeiten aufzeigt, beide Bereiche in Deutschland und in der Schweiz zu stärken und zukunftsfähig miteinander zu verknüpfen. Einen ersten konkreten Ansatzpunkt in der Forschung an dieser Schnittstelle liefert dazu die internationale Zusatzoption «Computational Thinking» zu ICILS 2018 (vgl. IEA 2016), an der sich u. a. Deutschland beteiligen wird.

\section{Literatur}

Aufenanger, Stefan. 1997. "Medienpädagogik und Medienkompetenz - Eine Bestandsaufnahme». In Enquete-Kommission «Zukunft der Medien in Wirtschaft und Gesellschaft. Deutschlands Weg in die Informationsgesellschaft), hrsg. v. Deutscher Bundestag, Medienkompetenz im Informationszeitalter, 15-22. Bonn: Deutscher Bundestag.

Bos, Wilfried, Birgit Eickelmann, und Julia Gerick. 2014. "Computer- und informationsbezogene Kompetenzen von Schülerinnen und Schülern der 8. Jahrgangsstufe in Deutschland im internationalen Vergleich». In ICILS 2013 - Computer- und informationsbezogene Kompetenzen von Schülerinnen und Schülern in der 8. Jahrgangsstufe im internationalen Vergleich, hrsg. v. Wilfried Bos, Birgit Eickelmann, Julia Gerick, Frank Goldhammer, Heike Schaumburg, Knut Schwippert, Martin Senkbeil, Renate Schulz-Zander und Heike Wendt, 113-145. Münster: Waxmann.

Bos, Wilfried, Birgit Eickelmann, Julia Gerick, Frank Goldhammer, Heike Schaumburg, Knut Schwippert, Martin Senkbeil, Renate Schulz-Zander, und Heike Wendt, Hrsg. 2014. ICILS 2013: computer- und informationsbezogene Kompetenzen von Schülerinnen und Schülern in der 8. Jahrgangsstufe im internationalen Vergleich. Münster: Waxmann.

Cohen, Jacob, Patricia Cohen, Stephan G. West, und Leona S. Aiken. 2013. Applied Multiple Regression/Correlation Analysis for the Behavioral Science. London: Lawrence Erlbaum Associates.

D-EDK. Deutschschweizer Erziehungsdirektoren-Konferenz. 2015. Schlussbericht zum Projekt Lehrplan 21.

Deutscher Bundestag. 18. Wahlperiode. 2015. Antrag der Fraktionen der CDU/CSU und SPD. Durch Stärkung der Digitalen Bildung Medienkompetenz fördern und digitale Spaltung überwinden. Berlin. http://dip21.bundestag.de/dip21/btd/18/044/1804422. pdf.

Döbeli Honegger, Beat. 2015. «Der lange Weg zum Klassenzimmer 2.0.» kommunalmagazin.ch: 24-28.

Eickelmann, Birgit, Wilfried Bos, Julia Gerick, und Julia Kahnert. 2014. «Anlage, Durchführung und Instrumentierung von ICILS 2013». In ICILS 2013: computer- und informationsbezogene Kompetenzen von Schülerinnen und Schülern in der 8. Jahrgangsstufe im internationalen Vergleich, hrsg. v. Wilfried Bos, Birgit Eickelmann, Julia Gerick, Frank Goldhammer, Heike Schaumburg, Knut Schwippert, Martin Senkbeil, Renate SchulzZander, und Heike Wendt, 43-81. Münster: Waxmann. 
Eickelmann, Birgit, Heike Schaumburg, Kerstin Drossel, und Renate Lorenz. 2014. «Schulische Nutzung von neuen Technologien in Deutschland im internationalen Vergleich». In ICILS 2013: computer- und informationsbezogene Kompetenzen von Schülerinnen und Schülern in der 8. Jahrgangsstufe im internationalen Vergleich, hrsg. v. Wilfried Bos, Birgit Eickelmann, Julia Gerick, Frank Goldhammer, Heike Schaumburg, Knut Schwippert, Martin Senkbeil, Renate Schulz-Zander, und Heike Wendt, 197-229. Münster: Waxmann.

Eickelmann, Birgit, Wilfried Bos, und Mario Vennemann. 2015. Total digital? - Wie Jugendliche Kompetenzen im Umgang mit neuen Technologien erwerben. Dokumentation der Analysen des Vertiefungsmoduls zu ICILS 2013. Münster: Waxmann.

Eickelmann, Birgit, Julia Gerick, und Wilfried Bos. 2014. «Die Studie ICILS 2013 im Überblick -Zentrale Ergebnisse und Entwicklungsperspektiven». In ICILS 2013 - Computer- und informationsbezogene Kompetenzen von Schülerinnen und Schülern in der 8. Jahrgangsstufe im internationalen Vergleich, hrsg. v. Wilfried Bos, Birgit Eickelmann, Julia Gerick, Frank Goldhammer, Heike Schaumburg, Knut Schwippert, Martin Senkbeil, Renate Schulz-Zander und Heike Wendt, 9-31. Münster: Waxmann.

Fletcher, Geoffrey, Dian Schaffhauser, und Douglas Levin. 2012. Out of Print: Reimagining the K-12 Textbook in a Digital Age. Washington, DC: State Educational Technology Directors Association (SETDA).

Fraillon, Julian, John Ainley, Wolfram Schulz, Tim Friedman, und Eveline Gebhardt. 2014. Preparing for life in a digital age. The IEA International Computer and Information Literacy Study International Report: Springer.

Fraillon, Julian, Wolfram Schulz, und John Ainley. 2013. International Computer and Information Literacy Study: Assessment framework. Amsterdam: IEA.

Fraillon, Julian, Wolfram Schulz, Tim Friedman, John Ainley, und Eveline Gebhardt. 2015. ICILS 2013 Technical Report. Amsterdam: IEA.

Gander, Walter, Antoine Petit, Gérard Berry, Barbara Demo, Jan Vahrenhold, Andrew McGettrick, Roger Boyle, Michéle Drechsler, Avi Mendelson, Chris Stephenson, Carlo Ghezzi, und Bertrand Meyer. 2013. Informatics education: Europe cannot afford to miss the boat ACM Europe: Informatics Education Report. New York.

Gl-Gesellschaft für Informatik. 2008. "Grundsätze und Standards für die Informatik in der Schule. Bildungsstandards für die Sekundarstufe I». LOG IN 28 (150/151): Beilage. https://www.gi.de/fileadmin/redaktion/empfehlungen/Bildungsstandards_2008.pdf.

Gl-Gesellschaft für Informatik. 2016. Dagstuhl-Erklärung: Bildung in der digital vernetzten Welt. https://www.gi.de/fileadmin/redaktion/Themen/dagstuhl-erklaerung-bildung-inder-digitalen-welt-2016.pdf.

IEA. 2016. Computational Thinking. Leaflet. Amsterdam: IEA.

Jung, Michael und Ralph Carstens. 2015. ICILS 2013 user guide for the international database. Amsterdam: IEA.

Konsortium icils.ch. 2015. Internationale Computer- und Informationskompetenzstudie (ICILS 2013). Schweiz First Findings. Konsortium icils.ch. Verfügbar unter: www.icils.ch.

Lorenz, Ramona, Julia Gerick, Renate Schulz-Zander, und Birgit Eickelmann. 2014. «Computer- und informationsbezogene Kompetenzen von Mädchen und Jungen im internationalen Vergleich». In ICILS 2013 - Computer- und informationsbezogene Kompetenzen von Schülerinnen und Schülern in der 8. Jahrgangsstufe im internationalen Vergleich, hrsg. v. Wilfried Bos, Birgit Eickelmann, Julia Gerick, Frank Goldhammer, Heike Schaumburg, Knut Schwippert, Martin Senkbeil, Renate Schulz-Zander und Heike Wendt, 231263. Münster: Waxmann. 
Schulz-Zander, Renate. 1998. "Lernen in der Informationsgesellschaft». In Schulkultur als Gestaltungsaufgabe, hrsg. v. Josef Keuffer, Heinz-Hermann Krüger, Sibylle Reinhardt, Elke Weise und Hartmut Wenzel, 407-422. Weinheim: Deutscher Studien Verlag.

Senkbeil, Martin, Frank Goldhammer, Wilfried Bos, Birgit Eickelmann, Knut Schwippert, und Julia Gerick. 2014. «Das Konstrukt der computer- und informationsbezogenen Kompetenzen in ICILS 2013». In ICILS 2013 - Computer- und informationsbezogene Kompetenzen von Schülerinnen und Schülern in der 8. Jahrgangsstufe im internationalen Vergleich, hrsg. v. Wilfried Bos, Birgit Eickelmann, Julia Gerick, Frank Goldhammer, Heike Schaumburg, Knut Schwippert, Martin Senkbeil, Renate Schulz-Zander und Heike Wendt, 83-112. Münster: Waxmann.

Tulodziecki, Gerhard. 1997. Medien in Erziehung und Bildung. Grundlagen und Beispiele einer handlungs- und entwicklungsorientierten Medienpädagogik (3. Aufl.). Bad Heilbrunn: Klinkhardt.

Wendt, Heike, Mario Vennemann, Knut Schwippert, und Kerstin Drossel. 2014. «Soziale Herkunft und computer- und informationsbezogene Kompetenzen von Schülerinnen und Schülern im internationalen Vergleich». In ICILS 2013. Computer- und informationsbezogene Kompetenzen von Schülerinnen und Schülern in der 8. Jahrgangsstufe im internationalen Vergleich, hrsg. v. Wilfried Bos, Birgit Eickelmann, Julia Gerick, Frank Goldhammer, Heike Schaumburg, Knut Schwippert, Martin Senkbeil, Renate Schulz-Zander, und Heike Wendt, 265-296. Münster: Waxmann.

\section{Abbildungen}

Abb. 1.: Leistungsdifferenzen in den computer- und informationsbezogenen Kompetenzen zwischen Schülerinnen und Schülern, die Informatik belegen, und denen, die das Fach nicht belegen (eigene Darstellung).

Abb. 2.: Differenzen in den computer- und informationsbezogenen Kompetenzen zwischen Schülerinnen und Schülern (Teilstichprobe der Schülerinnen und Schüler, die Informatik belegen), die Computer im Informatikunterricht nutzen und denen, die nie Computer im Informatikunterricht nutzen (eigene Darstellung). 


\section{Tabellen}

Tab. 1.: Angaben zur Frage: «Hast du in der Schule gelernt, wie die folgenden computerbasierten Tätigkeiten durchgeführt werden?», differenziert nach Schülerinnen und Schülern in Deutschland und in der Schweizer Stichprobe, die das Fach Informatik belegt haben, und denen, die das Fach nicht belegt haben (Angaben der Schülerinnen und Schüler in Prozent, dargestellte Kategorie ja; eigene Darstellung).

Tab. 2.: Angaben zur Frage: «Hast du in der Schule gelernt, wie die folgenden [computerbezogenen] Aufgaben durchgeführt werden?», differenziert nach Schülerinnen und Schülern (Teilstichprobe der Schülerinnen und Schüler, die Informatik belegt haben), die den Computer in Informatik nutzen, und denen, die keinen Computer im Informatikunterricht nutzen (Angaben der Schülerinnen und Schüler in Prozent, dargestellte Kategorie ja; eigene Darstellung).

Tab. 3.: Motive und Einstellungen zur Computernutzung, differenziert nach Schülerinnen und Schülern, die das Fach Informatik belegen, und denen, die das Fach nicht belegen (Angaben der Schülerinnen und Schüler in Prozent, Kategorie trifft zu; eigene Darstellung).

Tab. 4.: Motive und Einstellungen zur Computernutzung, Teilstichprobe der Schülerinnen und Schüler, die Informatik belegt haben, differenziert nach Jugendlichen, die den Computer im Informatikunterricht nutzen, und denen, die nie Computer im Informatikunterricht nutzen (Angaben der Schülerinnen und Schüler in Prozent, Kategorie trifft zu; eigene Darstellung).

Tab. 5.: Regressionsmodell zur Erklärung der computer- und informationsbezogenen Kompetenzen der Schülerinnen und Schüler in Deutschland (eigene Darstellung).

Tab. 6.: Regressionsmodell zur Erklärung der computer- und informationsbezogenen Kompetenzen der Schülerinnen und Schüler in der Schweizer Stichprobe ${ }^{1}$ (eigene Darstellung). 